\title{
SINGULAR VALUE DECOMPOSITION IN AHP
}

\author{
S. I. GASS ${ }^{1}$ and T. RAPCSÁK ${ }^{2}$ \\ ${ }^{1}$ Robert H. Smith School of Business, University of Maryland \\ College Park, Maryland 20472, USA \\ sgass@rhsmith.umd.edu \\ ${ }^{2}$ Laboratory of Operations Research and Decision Systems \\ Computer and Automation Institute, Hungarian Academy of Sciences, \\ P. O. Box 63, Budapest, Hungary \\ moczar@oplab.sztaki.hu
}

\begin{abstract}
The Analytic Hierarchy Process (AHP) (Saaty, 1990) has been accepted as a leading multiattribute decision model both by practitioners and academics. AHP can solve decision problems in various fields by the prioritization of alternatives. The heart of the most familiar version of the AHP is the Saaty's eigenvector method (EM) which approximates an $n \times n$ positive reciprocal matrix $A=\left(a_{i j}\right),\left(a_{i j}\right)=\frac{1}{a_{i j}}, i, j=1, \ldots, n$, , by a vector $\mathbf{w} \in R_{+}^{n}$, where $R_{+}^{n}$ is the positive orthant of the $n$-dimensional Euclidean space $R^{n}$, such that the matrix of the ratios $\left(w_{i} / w_{j}\right), i, j=1, \ldots, n$, is the best approximation to $A$, in some sense. It is emphasized that the EM results in a priority vector $\mathbf{w} \in R_{+}^{n}$ and an inconsistency number $\lambda$ max.

However, the EM has been criticised both from prioritization and consistency points of view and several new techniques have been developed.There are two different approaches in AHP: deterministic and statistical or stochastic.

In the deterministic approach, the underlying assumption is that one can observe the preferences with certainty and the only uncertainty lies in the elicitation of these preferences which give rise to the inconsistency condition. The EM belongs to the deterministic approach. Other approaches include the least-squares method (LSM) (Jensen, 1984), the chi-square method (CSM), (Jensen, 1984), the logarithmic least-squares method (LLSM) (Saaty and Vargas, 1984) and the weighted least-squares method (WLSM) (Chu et al., 1979). In the statistical approach, it is assumed that the preference judgments are random variables associated to an unknown probability distribution.

In [11], Saaty compares EM with LLSM and states that EM captures the inconsistency of dominance, while LLSM minimizes a distance function and searches for symmetry without an explicit attempt to capture dominance. But inconsistent dominance judgments are asymmetric. In this case, EM and LLSM give rise to different derived scales, and, at times, to a different choice. Moreover, he summarized ten reasons for not using LLSM instead of EM.

In [1], WLSM is compared with EM. WLSM has the advantage that it involves the solution of a system of linear algebraic equations and is, thus, conceptually easier to be understood than EM. Comparisons show that sums for WLSM are less than those for EM, and the dominant weight seems to be larger for WLSM.

Throughout Saaty's work only the right eigenvectors are used. However, theoretically, the use of left eigenvectors should be equally justified. This problem was observed first by Johnson et al. (1979). In [2], a new method, known as the Modified AHP (MAHP), claimed that the right and left eigenvectors inconsistency problem can be effectively reduced. In [13], an attempt was made to compare AHP and MAHP by using 42 models comprising 294 reciprocal matrices. It was revealed that MAHP is not better than AHP.
\end{abstract}


Singular value decomposition (SVD) is an important tool of matrix algebra that has been applied to a number of areas, for example, principal component analysis and canonical correlation in statistics, the determination of MoorePenrose generalized inverse, and low rank approximation of matrices, Kennedy and Gentle (1980), Eckart and Young (1936), Greenacre (1984). The matrix algebra and computational aspects of SVD are discussed in Kennedy and Gentle (1980), and statistical applications are described in Greenacre (1984). The aim of this lecture is to show that the SVD seems to be a good tool to provide a theoretically well-founded solution in AHP both for the scaling and consistency problems and to develop further the results of Gass and Rapcsák (1998) as well as Standard (2000) in order to derive the weight vector in a convenient and consistent way in AHP.

\section{References}

[1] Chu, A. T. W., Kalaba, R. E. and Spingarn, K., A comparison of two methods for determining the weights of belonging to fuzzy sets, Journal of Optimization Theory and Applications 4 (1979) 531-538.

[2] Donegan, H. A., Dodd, F. J. and McMaster, T. B. M., A new approach to AHP decision-making, The Statistician 41 (1992) 295-302.

[3] Eckart, C. and Young, G., The approximation of one matrix by another of lower rank, Psychometrika 1 (1936) 211-218.

[4] Gass, S. I. and Rapcsák, T., A note on synthesizing group decisions, Decision Support Systems 22 (1998) 5963.

[5] Greenacre, M. J., Theory and applications of correspondence analysis, Academic Press, London, Orlando, 1984.

[6] Jensen, R. E., An alternative scaling method for priorities in hierarchical structures, Journal of Mathematical Psychology 28 (1984) 317-332.

[7] Johnson, C. R., Beine, W. B. and Wang, T. J., Right-left asymmetry in an eigenvector ranking procedure, Journal of Mathematical Psychology 19 (1979) 61-64.

[8] Kennedy, W. J. and Gentle, J. E., Statistical computing, Marcel Dekker, New York, Basel, 1980.

[9] Saaty, T. L., and Vargas, L. G., Inconsistency and rank preservation, Journal of Mathematical Psychology 28 (1984) 205-214.

[10] Saaty, T. L., The analytic hierarchy process, Mc Graw-Hill, New York, 1980.

[11] Saaty, T. L., Eigenvector and logarithmic least squares, European Journal of Operational Research 48 (1990) 156-160.

[12] Standard, S. M., Analysis of positive reciprocal matrices, Master of Arts Thesis, University of Maryland, 2000.

[13] Tung, S. L. and Tang, S. L., A comparison of the Saaty's AHP and modified AHP for right and left eigenvector inconsistency, European Journal of Operational Research 106 (1998) 123-128. 